\title{
Downregulation of the transcription factor KLF4 is required for the lineage commitment of T cells
}

\author{
Xiaomin Wen ${ }^{1}$, Haifeng Liu ${ }^{1}$, Gang Xiao ${ }^{1}$, Xiaolong Liu ${ }^{1}$

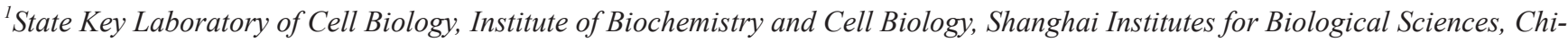 \\ nese Academy of Sciences, 320 Yueyang Road, Shanghai 200031, China
}

The roles of the reprogramming factors Oct4, Sox2, c-Myc and Klf4 in early T cell development are incompletely defined. Here, we show that Klf4 is the only reprogramming factor whose expression is downregulated when early thymic progenitors (ETPs) differentiate into T cells. Enforced expression of Klf4 in uncommitted progenitors severely impaired $T$ cell development mainly at the DN2-to-DN3 transition when $T$ cell lineage commitment occurs and affected the transcription of a variety of genes with crucial functions in early $\mathbf{T}$ cell development, including genes involved in microenvironmental signaling $(I L-7 R \alpha)$, Notch target genes (Deltex1), and essential T cell lineage regulatory or inhibitory genes (Bcl11a, SpiB, and IdI). The survival of thymocytes and the rearrangement at the Tcrb locus were impaired in the presence of enforced Klf4 expression. The defects in the DN1-to-DN2 and DN2-to-DN3 transitions in Klf4 transgenic mice could not be rescued by the introduction of a TCR transgene, but was partially rescued by restoring the expression of IL-7R $\alpha$. Thus, our data indicate that the downregulation of Klf4 is a prerequisite for $T$ cell lineage commitment.

Keywords: Klf4; early T cell development; T cell lineage commitment

Cell Research (2011) 21:1701-1710. doi:10.1038/cr.2011.183; published online 22 November 2011

\section{Introduction}

Mouse somatic cells can be reprogrammed into pluripotent stem cells by ectopic expression of the reprogramming factors Oct4, Sox2, c-Myc and Klf4, which are highly expressed in embryonic stem (ES) cells [1]. These four genes are the most powerful factors involved in the regulation of the transcriptional network that is necessary for the maintenance of pluripotency and selfrenewal of ES cells [2]. Presumably, the expression of these genes, especially Oct4, Sox 2 and Klf4 may need to be turned off or downregulated during the differentiation of pluripotent cells into a variety of differentiated derivatives.

Early $\mathrm{T}$ cell development is a multi-stage process in which progenitors gradually acquire $\mathrm{T}$ cell characteristics while losing their developmental plasticity or "stemness"

Correspondence: Xiaolong Liu

Fax: +86-21-54921178

E-mail: liux@sibs.ac.cn

Received 12 July 2011; revised 20 September 2011; accepted 17 October 2011; published online 22 November 2011
[3]. T cells are derived from lineage (Lin) ${ }^{-} \mathrm{Scal}^{+} \mathrm{Kit}^{+} \mathrm{Flt} 3$ hematopoietic stem cells (HSCs) that reside in the bone marrow (BM). HSCs progress to multipotent progenitors (MPPs) and lymphoid-primed MPPs (LMPPs), [4] and then differentiate into $\mathrm{Lin}^{-} \mathrm{Sca} 1{ }^{\text {low }} \mathrm{Kit}{ }^{\text {low }} \mathrm{Flt} 33^{\text {hi }} \mathrm{IL}-7 \mathrm{R} \alpha{ }^{\text {hi }}$ common lymphoid progenitors (CLPs). $\mathrm{CCR}^{+} \mathrm{CCR}^{+}$ LMPPs and CLPs enter the thymus via the blood circulation and become ETPs with multi-lineage potential to give rise to $\mathrm{T}$ cells, dendritic cells (DCs), myeloid cells, natural killer (NK) cells, and B cells [3]. As these progenitors receive signals, including Notch, c-Kit, and IL-7, from the thymic microenvironment [5], they initiate $\mathrm{T}$ cell differentiation and gradually lose their developmental potential for non- $\mathrm{T}$ cell lineages. $\mathrm{CD} 4^{-} \mathrm{CD} 8$ double negative (DN) thymic progenitors consist of DN1 $\left(\mathrm{CD} 44^{+} \mathrm{CD} 25^{-}\right)$, DN2 $\left(\mathrm{CD} 44^{+} \mathrm{CD} 25^{+}\right)$, DN3 $\left(\mathrm{CD} 44^{-} \mathrm{CD} 25^{+}\right)$, and $\mathrm{DN} 4\left(\mathrm{CD} 44^{-} \mathrm{CD} 25^{-}\right)$populations [6-7]. DN1 and DN2 cells retain both $\mathrm{T}$ cell and non-T cell lineage developmental potential [8], whereas final $\mathrm{T}$ cell lineage commitment occurs after an irreversible transition from DN2 to DN3 when most T cell-specific genes are upregulated to a peak level $[3,5]$. Thus, the differentiation of uncommitted thymic progenitors into $\mathrm{T}$ 
cells is a process that can be used to explore the potential relationship between reprogramming factors and differentiation.

The four reprogramming factors have been shown to participate individually in developmental processes other than reprogramming. For example, c-Myc is required for the proliferation of thymocytes at the preTCR checkpoint [9], and Klf4 is a key regulator of monocyte development [10], B-cell numbers and activation-induced B-cell proliferation [11], and quiescence of $\mathrm{CD} 8^{+} \mathrm{T}$ cells [12], and is required for the production of IL-17 [13]. Gene expression profiling studies have also shown that Klf4 is expressed in mouse and human thymocytes [14-15]. However, with the exception c-Myc, the roles of the reprogramming factors in early $\mathrm{T}$ cell lineage commitment are not well-defined.

In this study, we examined the expression patterns of the reprogramming factors in BM progenitors and thymocyte populations. Klf4 was the only reprogramming factor that was downregulated during the differentiation of ETPs into T cells. Enforced Klf4 expression in thymocytes led to impaired transcription of many pivotal genes that specify $\mathrm{T}$ cell lineage and resulted in the inhibition of thymocyte survival and Tcrb rearrangement. Moreover, the impaired $\mathrm{T}$ cell lineage commitment in Klf4 transgenic mice could not be rescued by the introduction of a TCR transgene, but could be rescued to a certain extent by restoring IL-7R $\alpha$ expression. Thus, downregu- lation of the transcription factor Klf4 is required for the lineage commitment of $\mathrm{T}$ cells.

\section{Results}

Expression of reprogramming factors during the differentiation of HSCs into T cells

We examined the mRNA levels of the reprogramming factors (Oct4, Sox2, c-Myc and Klf4) in BM progenitor and thymocyte populations. All four genes were highly expressed in ES cells, as previously reported [1]. Oct4 and Sox 2 mRNA levels became undetectable in HSCs and at later stages of differentiation (Figure 1A and 1B), whereas $c-M y c$ was expressed at higher levels at many differentiation stages, including in DN3 and DN4 cells, compared to ES cells (Figure 1C). This is in agreement with a previous report that c-Myc is required for preTCR-induced proliferation [9]. Interestingly, Klf4 expression was decreased in HSCs, CLPs and DN1 thymocytes compared to ES cells, and Klf4 levels continued to decrease during the transition from DN1 to DN2. Klf4 levels finally became undetectable in DN3 cells and at later stages of differentiation (Figure 1D), which specifically correlates with $\mathrm{T}$ cell lineage specification.

\section{Enforced expression of Klf4 in ETPs blocks T cell lineage commitment at the DN2-to-DN3 transition}

To investigate whether the downregulation of Klf4 is
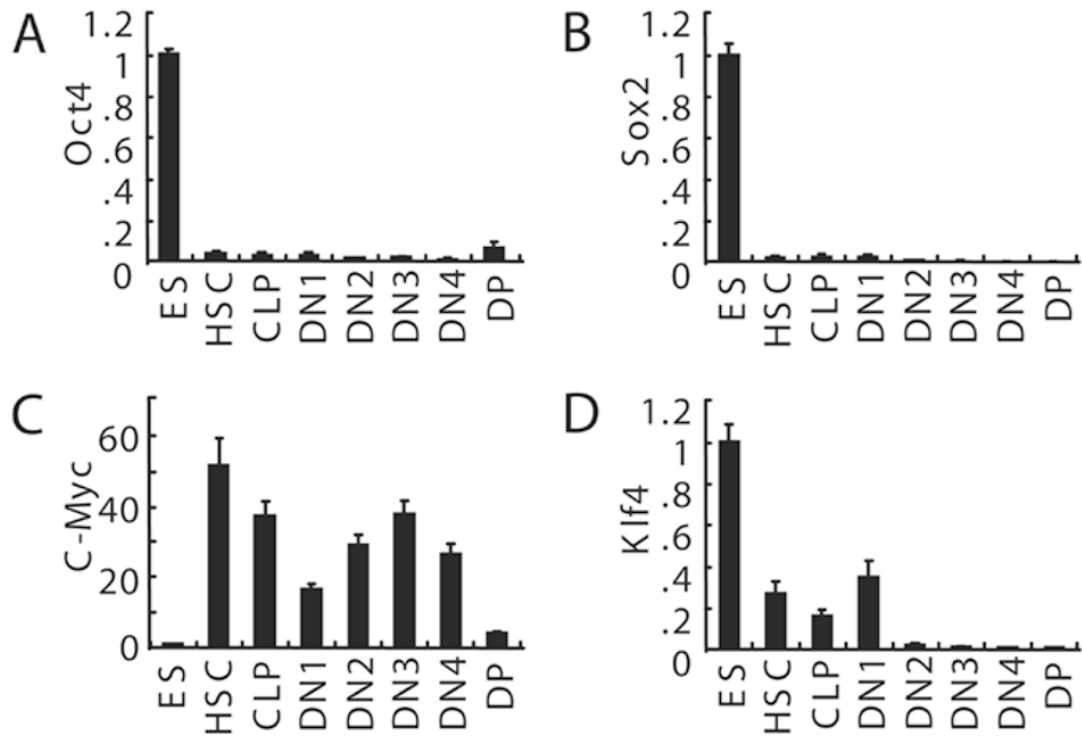

Figure 1 The expression profiles of Oct4, Sox2, c-Myc and Klf4 during the differentiation of HSCs into DP thymocytes. The ES cells were from a murine ES cell line E14 and the other indicated populations were sorted from wild-type mice. cDNA was prepared, and real-time PCR was performed to quantify the relative levels of transcripts of these four genes. For each gene, the values were normalized first to Gapdh transcript levels and then to ES levels, ES $=1$. Shown is the mean \pm SEM from three independent experiments. 
required for T-cell lineage specification, we generated Klf4 transgenic mice (Klf4Tg), in which Klf4 expression was driven by the human $\mathrm{CD} 2$ promoter and enhancer, al- lowing continuous expression of Klf4 in thymocytes at the DN1 (including ETPs and CD117'DN1 cells, Figure 2A) and subsequent differentiation stages (Figure 2B) [16].

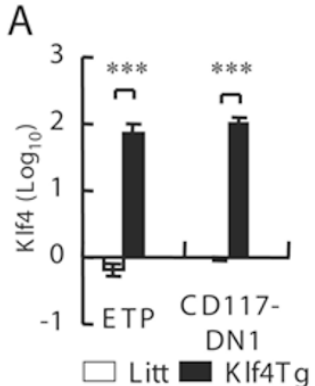

B

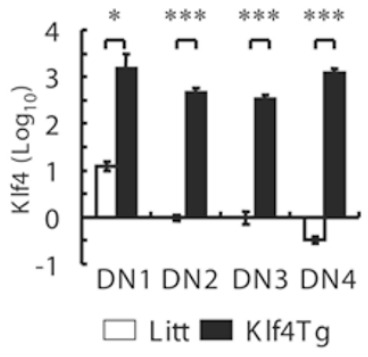

C
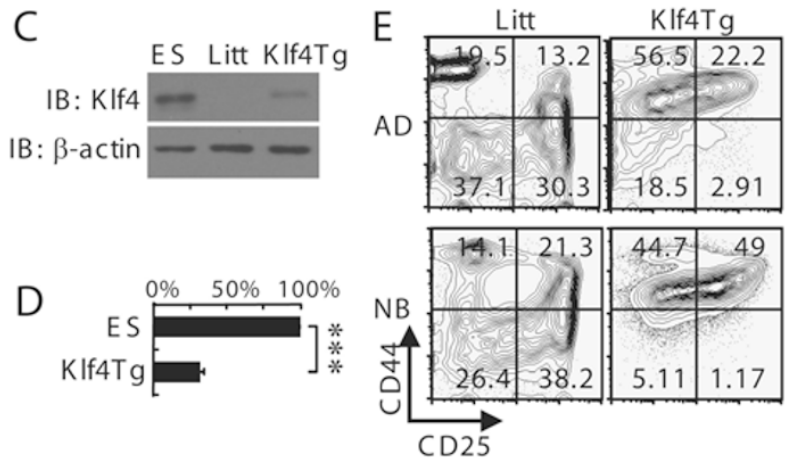

$\mathrm{F}$

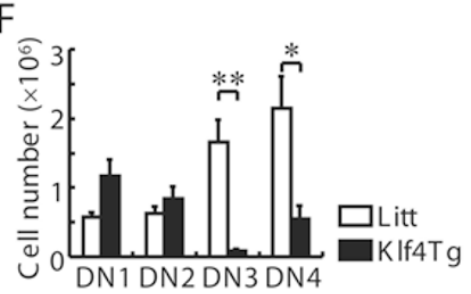

$\mathrm{H}$

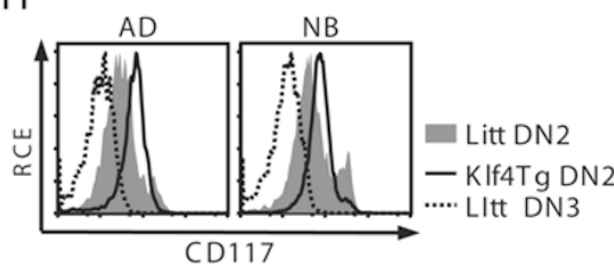

I
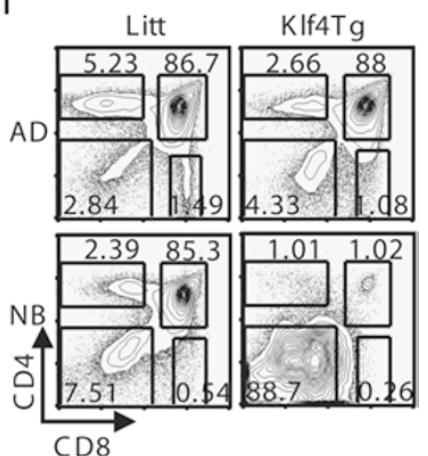

G

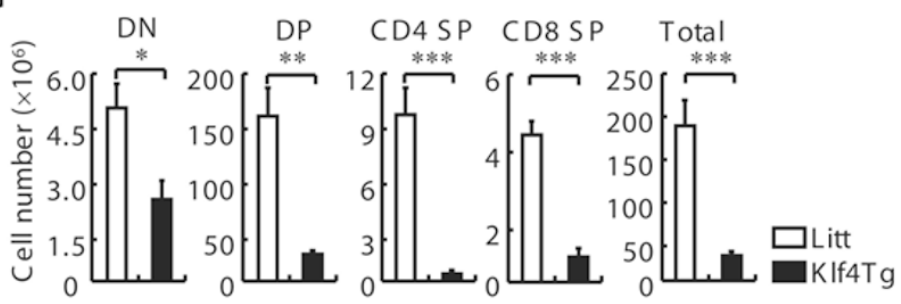

Figure 2 Enforced expression of Klf4 inhibits T cell lineage commitment mainly at the DN2-to-DN3 transition. (A) Both ETP and CD117 ${ }^{-}$DN1 populations have higher levels of KIf4 expression in transgenic mice. Real-time RT-PCR analysis for KIf4 was performed on ETP $\left(\mathrm{Lin}^{-} \mathrm{CD} 44^{+} \mathrm{CD} 25^{-} \mathrm{CD} 117^{+}\right.$, DN1a/b) and the other DN1 ( Lin $^{-} \mathrm{CD} 44^{+} \mathrm{CD} 25^{-} \mathrm{CD} 117^{-}$DN1c-e) populations purified from wild-type littermates (Litt) or Klf4Tg mice. The mean \pm SEM of $\log _{10}$-transformed data from four independent experiments are shown. Values were normalized to Litt CD117 ${ }^{-}$DN1 levels, Litt CD117 ${ }^{-}$DN1 $=\log _{10} 1=0$. (B) Real-time RT-

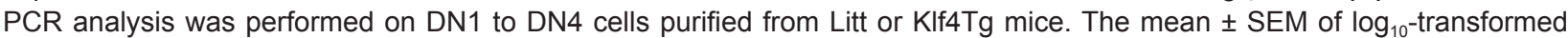
data from at least four independent experiments are shown. Values were normalized to Litt DN2 levels, Litt DN2 = $\log _{10} 1=0$. (C) Protein expression levels of KIf4 in ES cells (E14) and thymocytes from Litt and KIf4Tg mice. $\beta$-Actin was used as a loading control. (D) The bands of interest from ES cells (E14) and Klf4Tg thymocytes were further analyzed on a densitometer (Multi Gauge version 3.0 program). ${ }^{* * *} P<0.001$ compared to ES cells (mean \pm SEM, $n=4$ ). As Figure 1D has shown that the Klf4 transcript levels in DN1 were $\sim 35 \%$ of those in ES cells, we assume that the KIf4 protein levels in DN1 were also $\sim 35 \%$ of those in ES cells. Although the mRNA level of KIf4 was much higher in transgenic mice, the Klf4 protein levels in Klf4Tg thymocytes were $\sim 30 \%$ of those in ES cells (Figure 2), so the KIf4 protein levels in KIf4Tg thymocytes were comparable to the normal levels in DN1 cells. (E) Thymocytes from adult (AD) and newborn (NB) Litt or KIf4Tg mice were analyzed for Lin, CD44 and CD25 expression by flow cytometry. Gated Lin ${ }^{-}$DN cells were then analyzed for surface CD44 and CD25 expression. Representative flow cytometry profiles are shown. (F) Absolute numbers of DN1 to DN4 cells from Litt or Klf4Tg mice were calculated. Data are presented as the mean \pm SEM. $* P<0.05$; $* * P<0.01$; Litt $n=8$; KIf4Tg $n=7$. (G) Absolute cell numbers of total or DN, DP, CD4 SP and CD8 SP subpopulations from Litt or KIf4Tg mice were calculated. Shown is the mean \pm SEM. ${ }^{*} P<0.05 ; * * P<0.01 ; * * * P<0.001$. Litt $n=8$; Klf4Tg $n=7$. (H) The surface expression of CD117 on DN2 or DN3 cells from adult (AD) and newborn (NB) Litt or KIf4Tg mice. Relative cell number, RCE. Representative flow cytometry profiles are shown. (I) Enforced expression of Klf4 impairs T cell development in Klf4Tg mice. Thymocytes from adult (AD) and newborn (NB) Litt or Klf4Tg mice were analyzed for CD4 and CD8 expression by flow cytometry. The numbers next to or within the boxes of the contour diagrams indicate the percentage of cells. Representative flow cytometry profiles are shown. 
Five transgenic founders with similar phenotypes were generated and one line with Klf4 protein levels comparable to the normal levels in DN1 was used in this study (Figure 2C and 2D).

To examine the effects of continuous expression of Klf4 on T cell lineage specification, we analyzed DN cells after gating out cells expressing lineage markers (Lin: CD4, CD8a, CD3e, B220, Mac-1, Gr-1 and Ter119). In contrast to wild-type littermates (Litt), DN cells from Klf4Tg mice consisted of a very low percentage of DN3 and DN4 T-lineage-committed cells (Figure 2E). We also noticed that DN2 cells did not clearly separate from DN1 cells, and a DN1-DN2 transitional population characterized as $\operatorname{Lin}^{-} \mathrm{CD} 44^{+} \mathrm{CD} 25^{\text {low }}$ accumulated, which suggests a partial arrest at the DN1-to-DN2 transition in Klf4Tg mice. In terms of absolute numbers, Klf4Tg mice did not have reduced levels of DN1 and DN2 thymocytes compared with Litt mice, but DN3 thymocytes were dramatically reduced by 28 -fold (Figure $2 \mathrm{~F}$ ). Accordingly, the number of thymocytes at later stages of differentiation, including DN4, DP, CD4 SP and CD8 SP, and thus the total thymic cellularity was also significantly reduced (Figure $2 \mathrm{~F}$ and $2 \mathrm{G}$ ).

To further characterize DN2 thymocytes from Klf4Tg mice, we analyzed the surface expression of CD117 (cKIT), which is essential in the earliest precursors (DN1 and DN2) but is gradually downregulated at the DN3 stage [17-18]. As shown in Figure 2H, CD117 protein levels were higher in Klf4Tg DN2 thymocytes compared to DN2 or DN3 thymocytes from Litt mice, indicating a blockage of the DN2-to-DN3 transition.

To exclude the possibility that enforced expression of Klf4 "disguises" DN3 cells as DN2 cells by upregulating CD44 expression, the Klf4 transgene was introduced into mice on a $C D 44^{-1-}$ background [19]. We found that the generation of T-lineage-committed cells (DN3) remained impaired in Klf4Tg $C D 44^{--}$mice (Supplementary information, Figure S1A and S1B). We also observed a certain percentage of DP thymocytes in adult Klf4Tg mice (Figure 2I) and we questioned if this percentage is age-dependent in Klf4Tg mice. To this end, we analyzed thymocytes from newborn Litt and Klf4Tg mice. In contrast to the predominance of $\mathrm{CD} 4^{+} \mathrm{CD} 8^{+} \mathrm{DP}$ cells in Litt thymi, the thymi from newborn transgenic mice contained an overwhelming majority of DN cells (Figure 2I), which consisted only of uncommitted DN1 and DN2 thymic progenitors (Figure 2E). Moreover, DN2 thymocytes from newborn Klf4Tg mice also expressed higher levels of CD117 (Figure 2H). Thus, the impairment was mainly at the DN2-to-DN3 transition and not at the DNto-DP transition. This was clearly observed in newborn Litt and Klf4Tg mice; a small number of DN3 and later

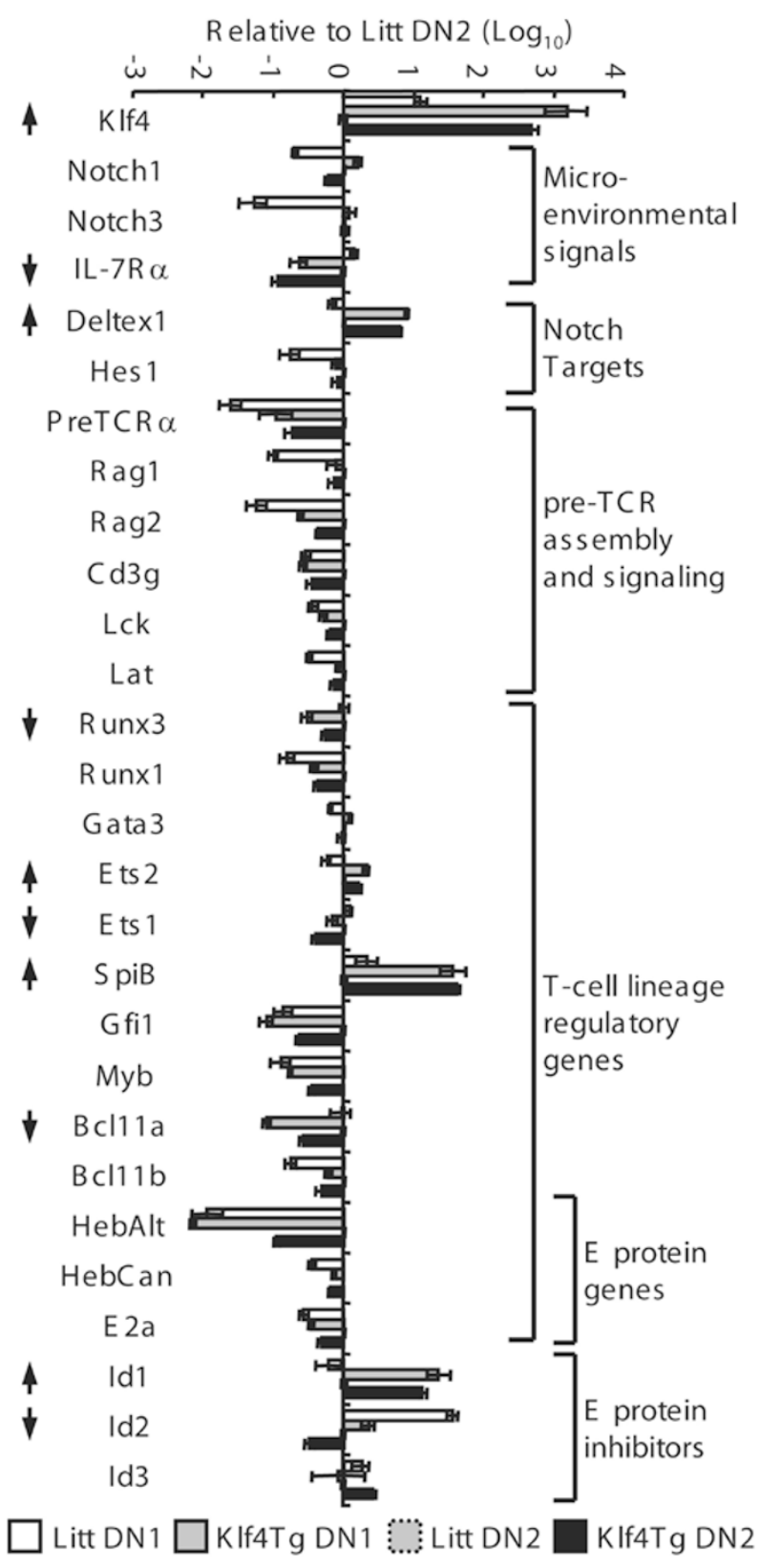

Figure 3 The transcription of a variety of pivotal genes that specify the $T$ cell lineage is altered in Klf4Tg mice. Real-time RT-PCR analysis was performed on DN1 ( Lin $^{-} \mathrm{CD} 44^{+} \mathrm{CD} 25^{-}$) and DN2 ( Lin $^{-} \mathrm{CD} 44^{+} \mathrm{CD} 25^{+}$) cells purified from Litt or KIf4Tg mice. The mean \pm SEM of $\log _{10}$-transformed data from four independent experiments are shown. For each gene, the values were normalized to Litt DN2 levels, Litt DN2 $=\log _{10} 1=0$. Up arrow, gene expression was significantly upregulated both in DN1 and DN2 cells in KIf4Tg mice compared to Litt; down arrow, the gene expression was significantly downregulated both in DN1 and DN2 cells in KIf4Tg mice compared to Litt.

stage (DN4, DP and SP) thymocytes accumulated with age, which led to a certain percentage of DP thymocytes 
in adult Klf4Tg mice. Taken together, these data suggest that enforced Klf4 expression in thymocytes severely impairs $\mathrm{T}$ cell development mainly at the DN2-to-DN3 transition during which $\mathrm{T}$ cell lineage commitment occurs.

The transcription of a variety of genes pivotal in specifying the T cell lineage is altered in Klf4Tg mice

To understand the molecular mechanism behind the inhibition of the DN1-to-DN2 or DN2-to-DN3 transition by continuous expression of Klf4, we purified DN1 and DN2 thymocytes, and analyzed the differences in gene expression between Klf4Tg and Litt mice. We examined the expression of genes that are well-known for their roles in differentiation and regulation of early T-cell development $[3,20]$, including genes involved in microenvironmental signaling, Notch target genes, pre-TCR assembly and signaling genes, and essential $\mathrm{T}$ cell lineage regulatory genes (Figure 3 ).

We observed that the expression of some T cellassociated genes, including preTCR $\alpha$, Rag2, Lat, Runx1, Bcll1b and HebCan, was higher in Klf4Tg DN1 and Klf4Tg DN2 cells than in Litt DN1 cells, but lower than in Litt DN2 cells. These characteristics are consistent with the Klf4Tg phenotype in which many DN1-DN2 transitional ( Lin $^{-} \mathrm{CD} 44^{+} \mathrm{CD} 25^{\text {low }}$ ) cells are inevitably included in the DN1 or DN2 population (Figure 2E). Remarkably, compared with Litt, there was a group of genes that exhibited similar changes in expression in both DN1 and DN2 cells from Klf4Tg mice; Deltex1, Ets 2, SpiB and $I d 1$ were upregulated, while $I L-7 R \alpha$, Runx3, Ets 1 , $B c l 11 a$ and Id2 were downregulated. Collectively, these results suggest that enforced expression of Klf4 inhibits $\mathrm{T}$ cell lineage commitment by affecting the transcription of these key genes.

\section{DN1 and DN2 cells do not deviate into other lineages in Klf4Tg mice}

Given that Klf4 may function in regulating the cell cycle of lymphocytes [11-12], we performed cell size and cell cycle analyses to determine whether enforced expression of Klf4 blocks the DN2-to-DN3 transition by inhibiting the proliferation of DN2 cells. As shown in Figure 4A, in Klf4Tg DN2 cells, the percentage of larger cells and cells in the $\mathrm{S} / \mathrm{G} 2 / \mathrm{M}$ phase of the cell cycle are not reduced. Thus, the proliferation of DN2 cells does not seem to be inhibited in Klf4Tg mice.

DN2 cells are able to differentiate into two different $T$ cell lineages, namely, $\alpha \beta$ T cells and $\gamma \delta$ T cells $[3,21]$. To determine if enforced expression of Klf4 also impairs the generation of $\gamma \delta \mathrm{T}$ cells, we performed flow cytometry analysis of $\mathrm{CD}^{-} \mathrm{CD}^{-} \mathrm{DN}$ cells and observed a reduction

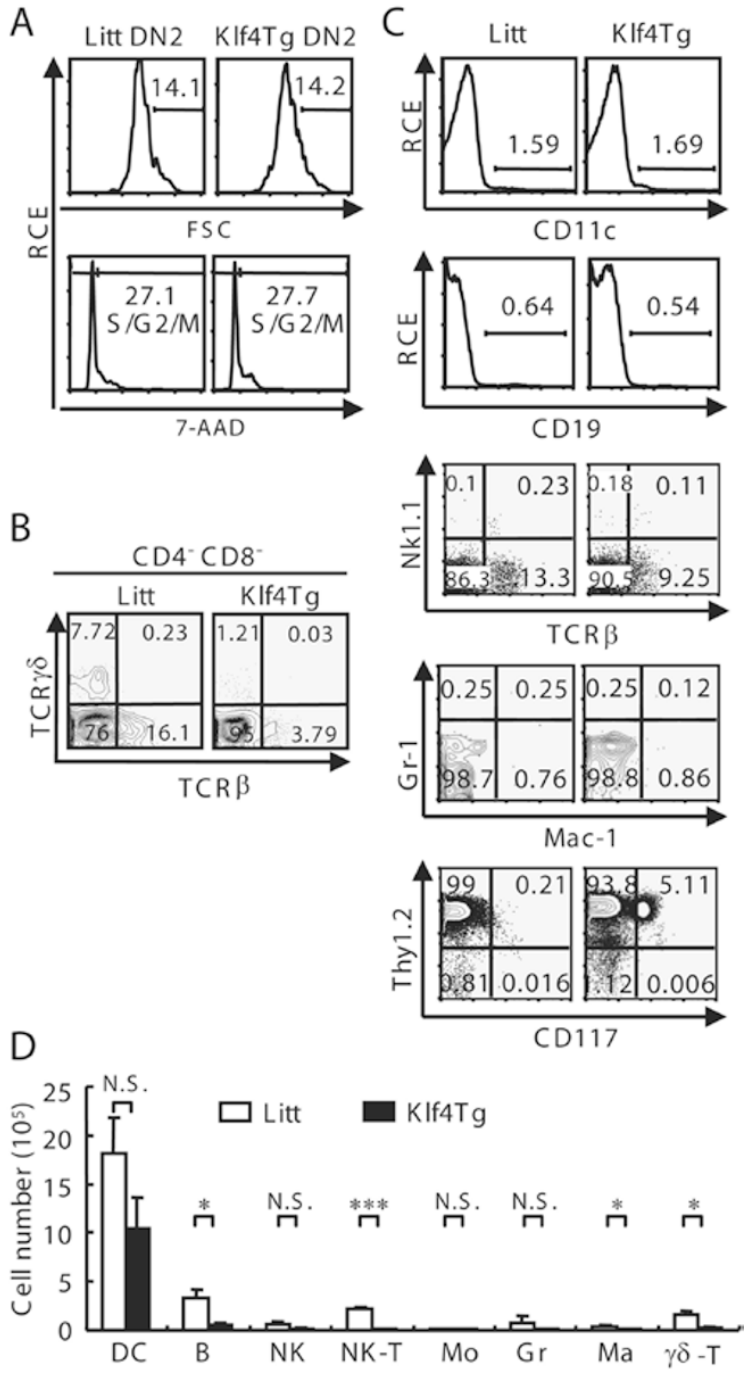

Figure 4 DN1 and DN2 cells proliferate normally and do not deviate into other lineages in Klf4Tg mice. (A) Cell size and cell cycle analyses of DN2 cells from littermates (Litt) or Klf4Tg mice. Cell size was compared using forward scatter (FSC) measurements (top) and cell cycle activity was determined by 7 -aminoactinomycin (7-AAD) staining (bottom). Relative cell number, RCE. Representative flow cytometry profiles are shown. (B) Surface expression of TCR $\beta$ and TCR $\gamma \delta$ was analyzed by flow cytometry for $\mathrm{CD}^{-} \mathrm{CD}^{-}$(DN) thymocytes from Litt or KIf4Tg mice. Data are representative of at least three independent experiments. (C) No significant accumulation of non-T lineage cells occurs in Klf4Tg thymi. Thymocytes from Litt or KIf4Tg mice were prepared and analyzed for markers of $B$ cells $\left(C D 19^{+}\right)$, NK cells $\left(\mathrm{NK} 1.1^{+} \mathrm{TCR} \beta^{-}\right)$, NK-T cells $\left(\mathrm{NK} 1.1^{+} \mathrm{TCR} \beta^{+}\right)$, monocytes $\left(\right.$ Thy $1.2^{-}$Mac $\left.-1^{+}\right)$, granulocytes $\left(\right.$Thy $\left.1.2^{-} \mathrm{Gr}-1^{+}\right)$, mast cells (Thy $\left.1.2^{-} \mathrm{CD} 117^{+}\right)$and DCs $\left(\mathrm{CD} 11 \mathrm{c}^{+}\right)$by flow cytometry analysis. Relative cell number, RCE. Representative flow cytometry profiles are shown. (D) Absolute cell numbers of DCs, B cells (B), NK cells (NK), NK-T cells (NK-T), monocytes (Mo), granulocytes $(\mathrm{Gr})$, mast cells (Ma) and $\gamma \delta \mathrm{T}$ cells from Litt or Klf4Tg mice were quantified by multiplying the total number of live cells by the fraction of cells in that population. Data are presented as the mean \pm SEM. $* P<0.05$; N.S. Not significant. Litt $n=8$; KIf4Tg $n=10$. 
of $\gamma \delta \mathrm{T}$ cells in Klf4Tg mice (Figure 4B and 4D). This suggests that enforced expression of Klf4 inhibits both $\alpha \beta$ and $\gamma \delta$ T cell lineage commitment. To further explore the fate of Klf4Tg DN1 and DN2 thymocytes, we analyzed thymocytes for markers of B cells, NK cells, NK-T cells, monocytes, granulocytes, mast cells and DCs by flow cytometry, but no significant augmentation of these non-T lineage cells was observed (Figure 4C and 4D). Taken together, these data indicate that Klf4Tg DN1 and DN2 cells do not differentiate into other lineages.

Thymocyte survival and Tcrb recombination are impaired in the presence of enforced Klf4 expression

We examined the survival of thymocytes and observed an increase in apoptosis in DN2 and DN3 cells from Klf4Tg mice (Figure 5A). Considering that the arrest at the DN2-to-DN3 transition might lead to an accumulation of DN2 cells, increased apoptosis could be the reason why Klf4Tg mice were able to retain similar DN2 cellularity as Litt mice, even though Klf4Tg DN2 thymocytes failed to differentiate further.

Pre-TCR signaling is necessary for DN3 cell survival [22], and rearrangement at the Tcrb locus is a hallmark of $\mathrm{T}$ cell lineage commitment [23]. As shown in Figure $5 \mathrm{~B}$ and $5 \mathrm{C}$, an impairment of $\mathrm{D} \beta 2-\mathrm{J} \beta 2$ and $\mathrm{V}-\mathrm{DJ} \beta$ rearrangements and a reduction of TCR $\beta$ intracellular protein levels in DN3 and DN4 cells from Klf4Tg mice were observed. Notably, the surface expression levels of TCR $\beta$ were lower in periphery $\mathrm{CD} 4^{+} \mathrm{T}$ or $\mathrm{CD} 8^{+} \mathrm{T}$ cells from Klf4Tg mice and the distribution of TCR V $\beta$ was somewhat altered in Klf4Tg mice (Supplementary information, Figure S2). Therefore, we introduced a functional TCR $\alpha \beta$ (OT-I) transgene into the Klf4Tg mice. As shown in Figure 5D and Supplementary information, Figure S3A-S3E, T cell development was not rescued. These results provided further validation that the main defect in Klf4Tg mice is at the DN1-to-DN2 and DN2-to-DN3 transition, which could not be rescued by the introduction of an OT- 1 transgene that promotes $\beta$-selection.

It has been reported that Notch, CD117 and IL-7 signaling in the thymic microenvironment are required for the survival and differentiation of DN1 and DN2 thymocytes [5, 17, 24-25]. While Klf4Tg DN2 thymocytes expressed even higher levels of CD117 (Figure 2H) and Notch1 expression was slightly reduced in Klf4Tg DN2 thymocytes, IL-7R $\alpha$ mRNA (Figure 3) and protein (Figure 6A) expression was severely impaired in Klf4Tg DN1 and DN2 thymocytes. Thus, Klf4Tg DN1 and DN2 thymocytes were infected with a retroviral construct encoding the Notch1 intracellular domain (NICD) or IL$7 \mathrm{R} \alpha$ and then transferred to fetal thymus organ culture (FTOC). After 2 days of culture, compared with vector-
A
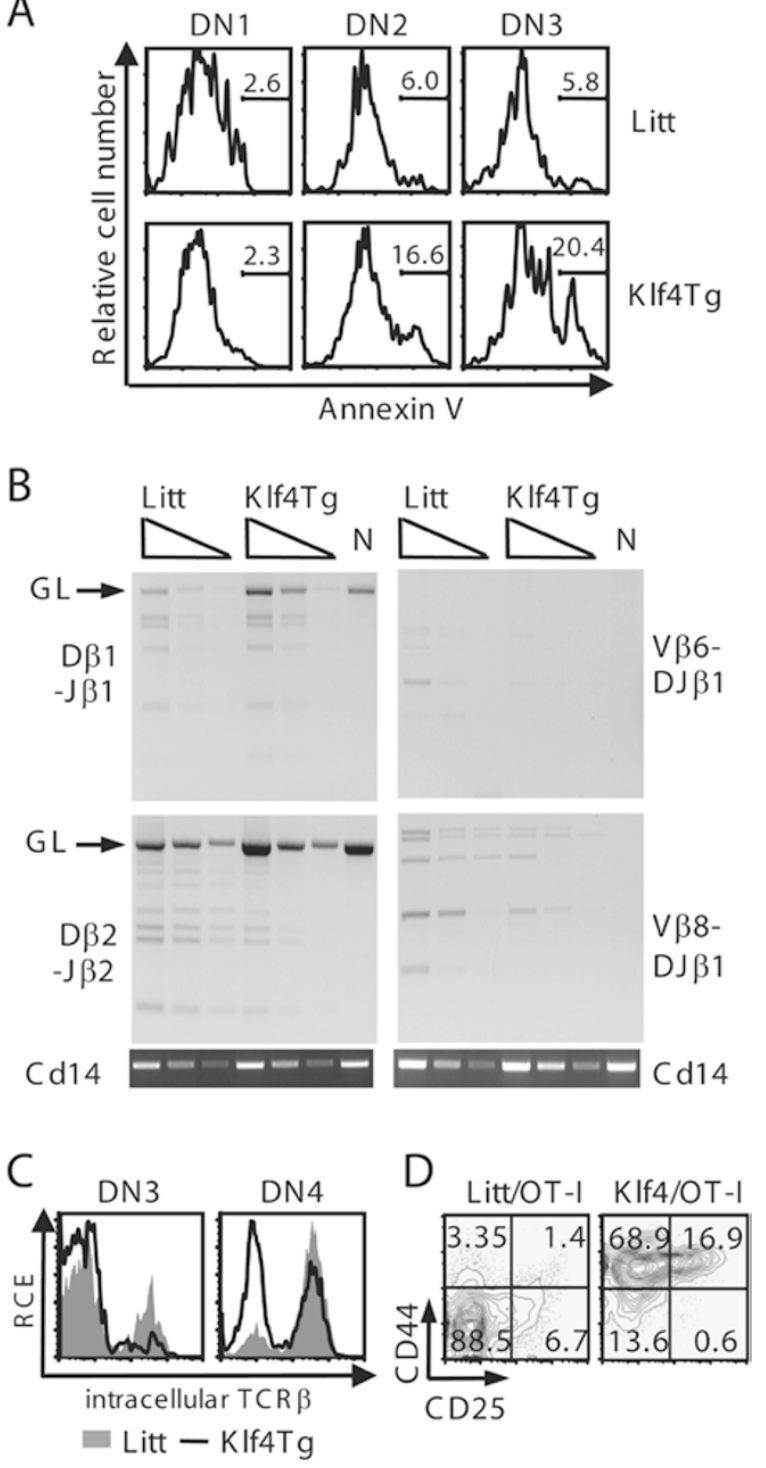

Figure 5 Enforced Klf4 expression inhibits the survival of T lineage cells and impairs the Tcrb rearrangement. (A) Apoptosis in DN1, DN2 and DN3 populations from Litt or KIf4Tg mice was measured by Annexin $\mathrm{V}$ staining after gating out propidium iodide positive cells. Representative flow cytometry profiles are shown. (B) Semi-quantitative PCR depicting the extent of $D \beta$ $J \beta$ and $V$-DJ $\beta$ rearrangements in DN thymocytes purified from KIf4Tg, Litt and Rag $1^{-/-}$mice (negative control, N) and analyzed by 3-fold serial dilution of genomic DNA [46]. Below, amplification of a Cd14 fragment as an input control. GL, germline fragment. The results are representative of two independent experiments. (C) Histograms showing intracellular staining of TCR $\beta$ in DN3 and DN4 cells from Litt or Klf4Tg mice. Relative cell number, RCE. Representative flow cytometry profiles are shown. (D) Thymocytes from Litt or KIf4Tg mice in the presence of the OT-I TCR transgene were prepared and analyzed for CD4, CD8, CD44 and CD25 expression by flow cytometry. Gated DN thymocytes were then analyzed for surface CD44 and CD25 expression. Representative flow cytometry profiles are shown. 
A

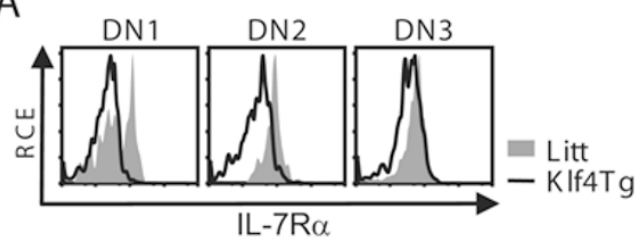

B

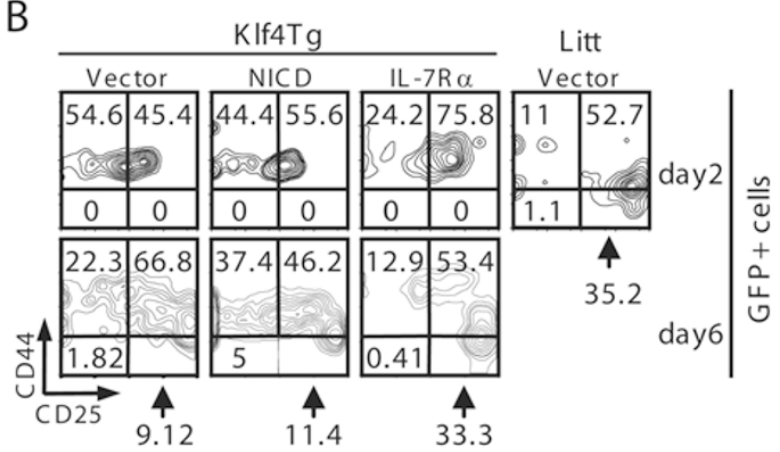

Figure 6 The Klf4Tg phenotype could be partially rescued by restoring the expression of IL-7R $\alpha$. (A) Thymocytes from Litt or KIf4Tg mice were prepared and analyzed for IL-7R $\alpha$, Lin, CD44 and CD25 expression by flow cytometry. Gated DN thymocytes were then analyzed for surface IL-7R $\alpha$ expression. Representative flow cytometry profiles are shown. (B) Klf4Tg DN1 and DN2 cell differentiation in FTOC. DN1 and DN2 cells from Litt or KIf4Tg mice were purified and infected with the pMCs-IRESGFP retrovirus encoding vector, NICD or IL-7R $\alpha$ and were then transferred to FTOC and cultured for the indicated number of days. The CD44/CD25 profiles of the GFP ${ }^{+}$fractions are shown. The above data are representative of at least three independent experiments.

infected Litt DN1 and DN2 cells (35.2\% of DN3, Figure $6 \mathrm{~B})$, neither NICD nor IL-7R $\alpha$ could rescue the differentiation of Klf4Tg DN1 and DN2 cells into DN3 cells, although there were higher percentages of DN2 cells in cells infected with NICD (55.6\%) or IL-7R $\alpha(75.8 \%)$ compared to those infected with vector $(45.4 \%)$. However, when the culture time was significantly increased (6 days, Figure 6B), IL-7R $\alpha$ promoted the differentiation of Klf4Tg DN1 and DN2 cells into DN3 cells (33.3\%) compared with vector-infected cells $(9.12 \%)$ to some extent. To summarize, enforced expression of Klf4 inhibited the survival of T lineage cells, and the defect in the DN1-toDN2 and DN2-to-DN3 transition in Klf4Tg thymocytes could be rescued to a certain extent by restoring the expression of IL-7R $\alpha$.

\section{Discussion}

The transcription factors Oct4, Sox2, c-Myc and Klf4 are four reprogramming factors that can recapture the pluripotency lost during differentiation by regulating the transcriptional network that controls the pluripotency and self-renewal of ES cells [26-27]. However, their roles in multipotent adult stem cells and specific lineage precursor cells are poorly understood. Our results show that $c-M y c$ is highly expressed, and Sox 2 and Oct4 expression are "turned off" in HSCs. Only Klf4 expression persists in the HSC stage and is finally "turned off" during T cell lineage specification. Enforced expression of Klf4 in thymocytes affects the transcription of many pivotal genes specifying $\mathrm{T}$ cell lineage, leading to increased apoptosis of $\mathrm{T}$ lineage cells, and inhibits $\mathrm{T}$ cell lineage commitment. Our studies here provide new insight into roles of reprogramming factors, especially Klf4, in early $\mathrm{T}$ cell development.

We have shown here that continuous expression of Klf4 in uncommitted progenitors severely impairs $\mathrm{T}$ cell lineage commitment. However, thymic progenitor DN1 cells express relatively high levels of Klf4 and downregulation of Klf4 is essential for normal $\mathrm{T}$ cell lineage commitment. Thus, our studies support the view that gene regulations during early $\mathrm{T}$ cell specification are mostly to downregulate prethymically inherited genes like Klf4, rather than to upregulate new T cell-specific factors [28-29]. Klf4 expression seems to be turned off at the DN1-to-DN2 transition. Enforced expression of Klf4 leads to an arrest at the DN1-to-DN2 transition, with the result that Klf4Tg DN2 cells are not bona fide DN2 cells, but closer to DN1-DN2 transitional ( $\operatorname{Lin}^{-} \mathrm{CD} 44^{+} \mathrm{CD} 25^{\text {low }}$ ) cells, which is supported by the gene expression characteristic analysis of DN1 and DN2 cells from Litt and Klf4Tg mice. The incompetence of DN2 cells leads to a block at the DN2-to-DN3 transition in Klf4Tg mice. In humans, NOTCH signaling downregulates KLF4 expression in the gut [30-31]. Interestingly, Notch signaling is also a positive regulator of $\mathrm{T}$ cell lineage and Notch ligands are found on stromal cells throughout the thymus $[5,32]$. These findings raise the possibility that Notch signals from the thymic microenvironment may negatively regulate Klf4 expression, which could in turn influence DN1 and DN2 cells to specify T cell lineage fate.

$T$ cell specification takes place under a tightly controlled transcriptional hierarchy. The E protein (also named helix-loop-helix protein) and Notch signaling cooperate to promote early T cell development [33]. HEBAlt, a long form of the E protein HEB gene, is specifically expressed in lymphoid precursor cells [34]. In thymic precursors, HEBAlt collaborates with Notch signals to promote early $\mathrm{T}$ cell development by suppressing $\mathrm{B}$ cell or myeloid potential [35-36]. $\mathrm{HEB}^{-/-} \mathrm{T}$ cells possess compromised Notch1 function and lose $\mathrm{T}$ cell potential [37]. The activity of E proteins is crucial for the DN2-to- 
DN3 transition and TCR gene rearrangement; loss of E2A leads to a reverse differentiation from DN3 cells to DN2 cells [38]. E proteins lose their function under high levels of Id1 protein, which can inhibit the DNA-binding activity of E proteins. It has been reported that Id1 transgenic mice expressed high levels of Detex 1, which inhibits the DN1-to-DN2 transition and leads to an accumulation of $\mathrm{CD} 4^{-} \mathrm{CD} 8^{-} \mathrm{CD} 44^{+} \mathrm{CD} 25^{\text {low }}$ cells [39]. This is similar to what is observed in Klf4Tg mice. Moreover, SpiB overexpression in DN2 thymocytes has been shown to lead to a blockage at the DN2-to-DN3 transition [40]. Thus, the defects in Klf4Tg mice may partly be caused by elevated levels of Id1 and SpiB or decreased levels of HEBalt.

$\mathrm{T}$ cell lineage specification is driven by signals unique to the thymic microenvironment [5]. IL-7 signaling is necessary to regulate the accessibility of the locus of TCR $\gamma$ [41] and this might explain the reduction of $\gamma \delta$ $\mathrm{T}$ cells in Klf4Tg mice, in which $I L-7 R \alpha$ expression is severely repressed. In addition, the IL-7/IL-7R pathway also plays a nonredundant role in early $\mathrm{T}$ cell development by facilitating survival, proliferation and TCR expression.[5, 42-45] Our results show that restoring the expression of IL-7R $\alpha$ could rescue, to some extent, the defects in $\mathrm{T}$ cell lineage commitment in Klf4Tg mice, raising the possibility that enforced expression of Klf4 inhibits $\mathrm{T}$ lineage cell survival by repressing the transcription of $I L-7 R \alpha$.

\section{Materials and Methods}

\section{Generation of Klf4 transgenic mice}

The Klf4 transgenic construct consisted of Klf4 cDNA driven by the human $\mathrm{CD} 2$ promoter and locus control region in the transgenic expression vector p29 22 (a gift from Paul Love). The transgenic mice were generated on a DBA/ $2 \times \mathrm{C} 57 \mathrm{BL} / 6$ background and then backcrossed to $\mathrm{C} 57 \mathrm{BL} / 6$ for at least ten generations. Transgene integrations of tail DNA were identified by PCR with the following primers. P1: 5'-TTGGCAAAGGAGCACATCAGA-3'; and P2: 5'-GTCTGGCAGGAAAGGAGGGTA-3'.

\section{Mice}

$C D 44^{-/-}$mice were obtained from the Model Animal Research Center, Nanjing University, China. C57BL/6 and OT-I TCR transgenic mice were obtained from Jackson Laboratory. Mice were analyzed between 4 and 8 weeks of age, except where otherwise indicated. All animal experiments were approved by the institutional animal use committee of the Shanghai Institutes for Biological Sciences, Chinese Academy of Sciences.

\section{Flow cytometric analysis, cell purification and antibodies}

Single-cell thymocyte or BM cell suspensions were prepared and stained as described [46]. Lineage negative cells were enriched by magnetic bead depletion using a lineage cocktail containing biotin-conjugated antibodies, anti-biotin beads and a MACS separator (Miltenyi Biotec). Individual subsets of Lin ${ }^{-}$thy- mocytes or BM cells were obtained by cell sorting using a FACSAria II cell sorter (BD Biosciences) for $\mathrm{Lin}^{-} \mathrm{Scal}^{+} \mathrm{Kit}^{+} \mathrm{Flt} 3^{-}$(HSC), Lin $^{-} \mathrm{Sca} 1^{\text {low }} \mathrm{Kit}^{\text {low }} \mathrm{Flt} 3^{\text {hi }} \mathrm{IL}-7 \mathrm{R} \alpha^{\text {hi }}$ (CLP), DN1 ( Lin $^{-} \mathrm{CD} 44^{+} \mathrm{CD} 25^{-}$), DN2 $\left(\mathrm{Lin}^{-} \mathrm{CD} 44^{+} \mathrm{CD} 25^{+}\right)$, DN3 ( $\left.\mathrm{Lin}^{-} \mathrm{CD} 44^{-} \mathrm{CD} 25^{+}\right)$and DN4 ( $\left.\mathrm{Lin}^{-} \mathrm{CD}^{-} 4^{-} \mathrm{CD} 25^{-}\right)$subsets. DP thymocytes were isolated by cell sorting $\mathrm{CD}_{4}^{+} \mathrm{CD} 8^{+}$. Aliquots of sorted cells were reanalyzed to ensure purity, which was greater than $90 \%$.

Antibodies (Abs) in lineage cocktails included Abs against CD11b, B220, CD4, CD8a, CD3e, Gr-1 and TER-119. Additional Abs used included Abs against CD44, CD25, TCR $\beta$, TCR $\gamma \delta$ and CD117. The Abs mentioned above were purchased from BD Pharmingen. Abs against IL7Ra, Sca1, Flt3 and Annexin V-FITC were from BioLegend. The anti-Klf4 (GKLF, H-180) Ab was from Santa Cruz Biotechnology.

\section{Real-time PCR}

Total RNA was extracted with TRIzol reagent (Invitrogen). Contaminating genomic DNA was removed from RNA samples with the DNA-Free kit (Ambion). Reverse transcription was performed using the SuperScript III First-Strand kit (Invitrogen). Real-time PCR was performed on a Rotor-Gene 6000 (Corbett Life Sciences) with the SYBR green real-time PCR master mix (Toyobo). Relative expression levels were normalized to Gapdh or $\beta$-actin transcript levels and calculated using the $2^{-\Delta \Delta C \mathrm{CT}}$ method. The sequences of the primers used have been previously published $[1,20,34,47-50]$ and are listed under Supplementary information, Table S1.

\section{Retroviral transduction and fetal thymic organ culture}

Murine cDNAs for the Notch 1 intracellular domain and $\mathrm{Il}$ $7 R \alpha$ were amplified and verified by sequencing, and then cloned into the pMCs-IRES-GFP retroviral vector. Retroviral transduction and FTOC were performed as described previously [40]. Briefly, sorted cells were infected, transduced with viral supernatant and seeded onto deoxyguanosine-depleted day 15 C57BL/6 host lobes in Terasaki microwell plate hanging drop cultures for 1 day, and the thymus lobes were then transferred to a freshly prepared filter/ sponge for regular thymus organ culture conditions.

\section{Statistical analysis}

Student's $t$-test was used for the comparison of two independent groups, and probability values of $P<0.05$ were considered significant. Statistical analyses of all numerical values in main figures are presented in Supplementary information, Figure S4.

\section{Acknowledgments}

The authors thank their colleagues Jinxiu Rui for helpful discussions, and Man Zhang and Jinsong Li for kindly providing ES cells. This work was supported in part by the Ministry of Science and Technology (2007CB815802 and 2012CB518700), the National Natural Science Foundation of China (30925031 and 30872290), and the Shanghai Municipal Government (09QH1402500).

\section{References}

1 Takahashi K, Yamanaka S. Induction of pluripotent stem cells from mouse embryonic and adult fibroblast cultures by defined factors. Cell 2006; 126:663-676. 
2 Kim J, Chu J, Shen X, Wang J, Orkin SH. An extended transcriptional network for pluripotency of embryonic stem cells. Cell 2008; 132:1049-1061.

3 Rothenberg EV, Moore JE, Yui MA. Launching the T-celllineage developmental programme. Nat Rev Immunol 2008; 8:9-21.

4 Adolfsson J, Månsson R, Buza-Vidas N, et al. Identification of Flt3+ lympho-myeloid stem cells lacking erythromegakaryocytic potential a revised road map for adult blood lineage commitment. Cell 2005; 121:295-306.

5 Petrie HT, Zúñiga-Pflücker JC. Zoned out: functional mapping of stromal signaling microenvironments in the thymus. Annu Rev Immunol 2007; 25:649-679.

6 Sitnicka E, Buza-Vidas N, Ahlenius H, et al. Critical role of FLT3 ligand in IL-7 receptor-independent T lymphopoiesis and regulation of lymphoid-primed multipotent progenitors. Blood 2007; 110:2955-2964.

7 Porritt HE, Rumfelt LL, Tabrizifard S, et al. Heterogeneity among DN1 prothymocytes reveals multiple progenitors with different capacities to generate $\mathrm{T}$ cell and non-T cell lineages. Immunity 2004; 20:735-745.

8 Taghon T, Yui MA, Rothenberg EV. Mast cell lineage diversion of $\mathrm{T}$ lineage precursors by the essential $\mathrm{T}$ cell transcription factor GATA-3. Nat Immunol 2007; 8:845-855.

9 Dose M, Khan I, Guo Z, et al. c-Myc mediates pre-TCR-induced proliferation but not developmental progression. Blood 2006; 108:2669-2677.

10 Feinberg MW, Wara AK, Cao Z, et al. The Kruppel-like factor KLF4 is a critical regulator of monocyte differentiation. EMBO J 2007; 26:4138-4148.

11 Klaewsongkram J, Yang Y, Golech S, Katz J, Kaestner KH, Weng NP. Kruppel-like factor 4 regulates B cell number and activation-induced B cell proliferation. J Immunol 2007; 179:4679-4684.

12 Yamada T, Park CS, Mamonkin M, Lacorazza HD. Transcription factor ELF4 controls the proliferation and homing of CD8(+) T cells via the Kruppel-like factors KLF4 and KLF2. Nat Immunol 2009; 10:618-626.

13 Lebson L, Gocke A, Rosenzweig J, et al. Cutting edge: the transcription factor Kruppel-like factor 4 regulates the differentiation of Th17 cells independently of ROR $\gamma \mathrm{t}$. J Immunol 2010; 185:7161-7164.

14 Tabrzifard S, Olaru A, Plotkin J, Fallahi-Sichani M, Livak F, Petrie HT. Analysis of transcription factor expression during discrete stages of postnatal thymocyte differentiation. $J$ Immunol 2004; 173:1094-1102.

15 Dik WA, Pike-Overzet K, Weerkamp F, et al. New insights on human $\mathrm{T}$ cell development by quantitative $\mathrm{T}$ cell receptor gene rearrangement studies and gene expression profiling. $J$ Exp Med 2005; 201:1715-1723.

16 Boer Jd, Williams A, Skavdis G, et al. Transgenic mice with hematopoietic and lymphoid specific expression of Cre. Eur J Immunol 2003; 33:314-325.

17 Massa S, Balciunaite G, Ceredig R, Rolink AG. Critical role for c-kit (CD117) in T cell lineage commitment and early thymocyte development in vitro. Eur J Immunol 2006; 36:526532.

18 Ceredig R, Rolink T. A positive look at double-negative thymocytes. Nat Rev Immunol 2002; 2:888-897.
19 Graham VA, Marzo AL, Tough DF. A role for CD44 in T cell development and function during direct competition between CD44+ and CD44- cells. Eur J Immunol 2007; 37:925-934.

20 Franco CB, Scripture-Adams DD, Proekt I, et al. Notch/Delta signaling constrains reengineering of pro-T cells by PU.1. Proc Natl Aca Sci USA 2006; 103:11993-11998.

21 Rothenberg EV, Taghon T. Molecular genetics of T cell development. Annu Rev Immunol 2005; 23:601-649.

22 Michie AM, Zuniga-Pflucker JC. Regulation of thymocyte differentiation: pre-TCR signals and beta-selection. Semin Immunol 2002; 14:311-323.

23 Dik WA, Pike-Overzet K, Weerkamp F, et al. New insights on human $\mathrm{T}$ cell development by quantitative $\mathrm{T}$ cell receptor gene rearrangement studies and gene expression profiling. $J$ Exp Med 2005:1715-1723.

24 Kim K, Lee CK, Sayers TJ, Muegge K, Durum SK. The trophic action of IL-7 on pro-T cells: inhibition of apoptosis of pro-T1, -T2, and -T3 cells correlates with Bcl-2 and Bax levels and is independent of Fas and p53 pathways. $J$ Immunol 1998; 160:5735-5741.

25 Ciofani M, Zuniga-Pflucker JC. A survival guide to early T cell development. Immunol Res 2006; 34:117-132.

26 Pei D. Regulation of pluripotency and reprogramming by transcription factors. J Biol Chem 2009; 284:3365-3369.

27 Yamanaka S. A fresh look at iPS cells. Cell 2009; 137:13-17.

28 David-Fung E-S, Butler R, Buzi G, et al. Transcription factor expression dynamics of early T-lymphocyte specification and commitment. Dev Biol 2009; 325:444-467.

29 Kawazu M, Yamamoto G, Yoshimi M, et al. Expression profiling of immature thymocytes revealed a novel homeobox gene that regulates double-negative thymocyte development. J Immunol 2007; 179:5335-5345.

30 Ghaleb AM, Aggarwal G, Bialkowska AB, Nandan MO, Yang VW. Notch inhibits expression of the Kruppel-like factor 4 tumor suppressor in the intestinal epithelium. Mol Cancer Res 2008; 6:1920-1927.

31 Real PJ, Tosello V, Palomero T, et al. Gamma-secretase inhibitors reverse glucocorticoid resistance in T cell acute lymphoblastic leukemia. Nat Med 2009; 15:50-58.

32 Rothenberg EV, Scripture-Adams DD. Competition and collaboration: GATA-3, PU.1, and Notch signaling in early T-cell fate determination. Semin Immunol 2008; 20:236-246.

33 Ikawa T, Kawamoto H, Goldrath AW, Murre C. E proteins and Notch signaling cooperate to promote T cell lineage specification and commitment. $J$ Exp Med 2006; 203:1329-1342.

34 Dionne CJ, Tse KY, Weiss AH, et al. Subversion of T lineage commitment by PU.1 in a clonal cell line system. Dev Biol 2005; 280:448-466.

35 Wang D, Claus CL, Rajkumar P, et al. Context-dependent regulation of hematopoietic lineage choice by HEBAlt. $J$ Immunol 2010; 185:4109-4117.

36 Braunstein M, Rajkumar P, Claus CL, et al. HEBAlt enhances the T-cell potential of fetal myeloid-biased precursors. Int Immunol 2010; 22:963-972.

37 Braunstein M, Anderson MK. HEB-deficient T-cell precursors lose T-cell potential and adopt an alternative pathway of differentiation. Mol Cell Biol 2011; 31:971-982.

38 Wojciechowski J, Lai A, Kondo M, Zhuang Y. E2A and HEB are required to block thymocyte proliferation prior to pre- 
TCR expression. J Immunol 2007; 178:5717-5726.

39 Wang HC, Perry SS, Sun XH. Id1 attenuates Notch signaling and impairs T cell commitment by elevating Deltex1 expression. Mol Cell Biol 2009; 29:4640-4652.

40 Lefebvre JM, Haks MC, Carleton MO, et al. Enforced expression of Spi-B reverses T lineage commitment and blocks betaselection. J Immunol 2005; 174:6184-6194.

41 Durum SK, Candeias S, Nakajima H, et al. Interleukin 7 receptor control of $\mathrm{T}$ cell receptor gamma gene rearrangement: role of receptor-associated chains and locus accessibility. $J$ Exp Med 1998; 21:2233-2241.

42 Yu Q, Erman B, Park JH, Feigenbaum L, Singer A. IL-7 receptor signals inhibit expression of transcription factors TCF1, LEF-1, and RORgammat: impact on thymocyte development. J Exp Med 2004; 200:797-803.

43 Peschon JJ, Morrissey PJ, Grabstein KH, et al. Early lymphocyte expansion is severely impaired in interleukin 7 receptordeficient mice. J Exp Med 1994; 180:1955-1960.

44 Gonzalez-Garcia S, Garcia-Peydro M, Martin-Gayo E, et al. CSL-MAML-dependent Notch1 signaling controls T lineagespecific IL-7R \{alpha\} gene expression in early human thymopoiesis and leukemia. J Exp Med 2009; 206:779-791.
45 Saba I, Kosan C, Vassen L, Möröy T. IL-7R-dependent survival and differentiation of early T-lineage progenitors is regulated by the $\mathrm{BTB} / \mathrm{POZ}$ domain transcription factor Miz-1. Blood 2011; 117:3370-3381.

46 Wang X, Xiao G, Zhang Y, et al. Regulation of Tcrb recombination ordering by c-Fos-dependent RAG deposition. Nat Immunol 2008; 9:794-801.

47 Agata Y, Tamaki N, Sakamoto S, et al. Regulation of T cell receptor beta gene rearrangements and allelic exclusion by the helix-loop-helix protein, E47. Immunity 2007; 27:871-884.

48 Taghon T, Yui MA, Pant R, Diamond RA, Rothenberg EV. Developmental and molecular characterization of emerging beta- and gammadelta-selected pre-T cells in the adult mouse thymus. Immunity 2006; 24:53-64.

49 Tydell CC, David-Fung ES, Moore JE, Rowen L, Taghon T, Rothenberg EV. Molecular dissection of prethymic progenitor entry into the T lymphocyte developmental pathway. J Immunol 2007; 179:421-438.

50 Yui MA, Rothenberg EV. Deranged early T cell development in immunodeficient strains of nonobese diabetic mice. $J$ Immunol 2004; 173:5381-5391.

(Supplementary information is linked to the online version of the paper on the Cell Research website.) 